\title{
PREPARATION AND CHARACTERIZATION OF POLY(L-LACTIC ACID) FILMS PLASTICIZED WITH GLYCEROL AND MALEIC ANHYDRIDE
}

\author{
Hikmatun Ni'mah ${ }^{1 *}$, Rochmadi Rochmadi², Eamor M. Woo ${ }^{3}$, Dian A. Widiasih ${ }^{1}$, \\ Siska Mayangsari ${ }^{1}$ \\ ${ }^{1}$ Department of Chemical Engineering, Faculty of Industrial Technology, Institut Teknologi Sepuluh \\ Nopember, Kampus ITS Sukolilo, Surabaya 60111, Indonesia \\ ${ }^{2}$ Department of Chemical Engineering, Faculty of Engineering, Gadjah Mada University, Jl. Grafika \\ No.2, Yogyakarta 55281, Indonesia \\ ${ }^{3}$ Department of Chemical Engineering, College of Engineering, National Cheng Kung University,
} Tainan 701, Taiwan

(Received: July 2018 / Revised: October 2018 / Accepted: April 2019)

\begin{abstract}
In this study, poly(L-lactic acid) (PLLA) was blended with glycerol as a plasticizer by the solution blending technique to form blend films. The glycerol content was varied in order to evaluate the effect of glycerol content on the PLLA properties and to obtain an optimum weight ratio of PLLA/glycerol (PLLA/Gly) blend films with improved properties. The effect of the addition of compatibilizer on the properties of the composite films was also observed. The properties of the films obtained were characterized by using FTIR, XRD, DMA and SEM. The FTIR spectra showed an increase in the intensity of the characteristic peak of glycerol with increasing glycerol content, indicating that the blending ratio and technique were precise. Based on the XRD analysis, the degree of crystallinity generally increased with the addition of glycerol. DMA analysis showed that the addition of glycerol reduced the value of tensile strength and Young's modulus of the PLLA/Gly films, but increased the elongation at break. The optimum weight ratio was reached by the sample of PLLA/Gly (80/20) with the value of tensile strength, Young's modulus and elongation at break being $13.43 \mathrm{MPa}, 747.8 \mathrm{MPa}$ and $1.96 \%$, respectively. The addition of compatibilizer slightly increased the flexibility of the composite films. DSC analysis showed an increase in flexibility after the addition of glycerol, indicated by a decrease in $\mathrm{T}_{\mathrm{g}}$, which supports the results of the DMA analysis. SEM analysis was made of the porous morphology on the fracture surface of the films after the addition of glycerol; the porous structure was more pronounced in the PLLA/Gly (80/20) film with compatibilizer, which could therefore be considered for application as a scaffold in tissue engineering after further analysis has been conducted.
\end{abstract}

Keywords: Blend; Glycerol; Maleic anhydride; Plasticizer; Poly(L-lactic acid)

\section{INTRODUCTION}

Poly(L-lactic acid) (PLLA) has become an interesting material in some specific applications due to its excellent properties, such as biocompatibility, biodegradability, high mechanical properties and processability. However, PLLA still has some drawbacks, such as brittleness due to its high crystallinity, hydrophobicity, and low thermal stability, which lead to limitations in its utilization. One of the approaches to overcome these drawbacks is by combining PLLA

\footnotetext{
*Corresponding author's email: hikmatun_n@chem-eng.its.ac.id, Tel. +62-31-5946240, Fax.+62-31-5999282 Permalink/DOI: https://doi.org/10.14716/ijtech.v10i3.2936
} 
with other materials to improve its properties or to generate new properties for a target application without developing new materials. Among several methods of material modification, the blending method, either solvent blending or melt blending, is simple and straightforward when compared to the polymerization method for copolymer formation. Some studies have reported blends of PLLA and other materials, such as blends of PLLA with other crystalline polymers, i.e. PLLA with poly(ethylene oxide (PEO) (Lee et al., 2012); PLLA with poly(D-lactic acid) (PDLA) (Ni'mah et al., 2014); PLLA with other amorphous polymers, i.e. PLLA with atactic poly(methyl methacrylate) (aPMMA) (Woo et al., 2014); PLLA with poly(vinyl phenol) (PVPh) (Ni'mah et al., 2014); PLLA with elastomeric polymers such as natural rubber (Desa et al., 2016; Nofar et al., 2019); PLLA with nanoparticles (Raquez et al., 2013); PLLA with biodegradable and biomaterial such as chitosan (Duarte et al., 2010) or cellulose (Ni'mah et al., 2017); and PLLA and plasticizers (Sitompul et al., 2016). All those modifications were made to meet the specifically desired properties for intended applications. PLLA has already been widely utilized in many applications, such as packaging, and in environmental and biomedical fields (Nofar et al. 2019).

Plasticizers have been reported to have been blended with PLLA to improve its mechanical properties (Sitompul et al., 2016). Plasticizers are additive materials that can increase the flexibility and durability of a material. For certain issues and applications, biodegradable plasticizers have become favored for use as PLLA modifiers. In biomedical applications, various PLLA blends have been investigated for application in drug delivery, implants, surgical sutures, orthopaedic devices and scaffolds in tissue engineering (Saini et al., 2016). For the last of these applications, some of the aspects that should be considered in using polyesters and their composites in tissue engineering are biodegradability, biocompatibility and morphology, as well as processability and the mechanical properties of the materials (Pavia et al., 2012). Some studies have reported the blending of PLLA with biopolymers, which have a plasticizing effect for biomedical applications. Chen et al. (2015) report the preparation of a porous scaffold from blend of PLLA and poly(ethylene glycol) (PEG) by supercritical $\mathrm{CO}_{2}$ foaming and particle leaching. The addition of PEG to the PLLA matrix has a plasticizing effect on the PLLA, indicated by the decrease in $T_{g}$ in it. Another study by Frydrych et al. (2015) showed that blends of PLLA and poly(glycerol sebacate) (PGS) provide a porous microstructure, good hydrophilic characteristics, and good mechanical properties, which have potential for application as scaffolds in tissue engineering. PGS is a biodegradable and biocompatible synthetic elastomer, which shows mechanical behavior resembling the properties of soft tissue (Wang et al., 2002; Rai et al., 2012). PGS is also a non-toxic material and contains monomers of glycerol.

In blending two or more components, one of the problems that should be tackled is the low compatibility between two or more components, which will influence the physical properties of the composites. To enhance this compatibility, the addition of a compatibilizer is a simple approach that can be taken. The compatibilizer used can be one or all the components that have been surface-modified by the grafting technique. Wang et al. (2012) report that composites of MA-grafted PLLA and cellulose acetate have good mechanical properties and are biocompatible. MA is a non-toxic material which has been used in the biomedical field. In addition, for wood-plastic composites (blends of polypropylene and polyethylene), grafting MA onto the composite plastics has led to improvements in the mechanical properties of the composites because of the enhancement of interfacial bonding and dispersion of wood in the matrix (Gao et al., 2012).

In this study, glycerol (Gly) as a plasticizer was added to PLLA to improve its properties, including an increase in its flexibility and strength. The selected plasticizer is a miscible, biocompatible and biodegradable material. PLLA grafted with maleic anhydride (MA) (PLLA- 
g-MA) was also added to the PLLA/Gly blend as a compatibilizer. In the molecular structure of the composites, the addition of MA was expected to be able to increase the number of carbonyl groups that would perform specific bonding with other components, so that it could be used as a compatibilizer (Zhang \& Sun, 2004). Therefore, the effects of the addition of PLLA-g-MA as a compatibilizer on the properties of the PLLA and PLLA/Gly blends were also investigated. To the best of our knowledge, no information is provided in the literature about the modification of PLLA with glycerol and PLLA-g-MA, so this study will contribute to research development in the polymer field.

\section{METHODS}

\subsection{Materials}

Poly(L-lactic acid) (PLLA) with 98\% L-form and $\mathrm{Mw}$ of 166,000 Kg/Kmol (measured by GPC) was obtained from Zhejiang Hisun Biomaterials Co., Ltd (China). Glycerol (Gly) as a plasticizer was supplied by Merck, Germany. All the materials were used as received, without any further purification.

\subsection{Sample Preparation}

For the preparation of the films, the PLLA and glycerol mixture was dissolved in chloroform at $60^{\circ} \mathrm{C}$ for \pm 6 hours under stirring to form a homogeneous solution with a concentration of 5 wt $\%$. The mixture solution was then poured into a glass Petri dish and the solvent allowed to evaporate at room temperature. The PLLA/Gly blend films obtained were further dried in an oven to evaporate the remaining solvent. The weight ratio of PLLA to Gly varied from 0-20 $\mathrm{wt} \%$ of glycerol with intervals of $5 \mathrm{wt} \%$.

The procedure for the PLLA-g-MA preparation is as follows. PLLA was grafted onto MA in reaction conditions of $60^{\circ} \mathrm{C}$ for 6 hours under stirring, with inert nitrogen $\left(\mathrm{N}_{2}\right)$ with xylene as a solvent and benzoic peroxide (BP) as an initiator. The grafting product was then purified by dissolving it in xylene, followed by filtering of the product. The powder on the surface of the filter was then washed with acetone and dried in a vacuum oven at $80^{\circ} \mathrm{C}$ for 24 hours. The composite films with the addition of PLLA-g-MA compatibilizer were prepared in a procedure similar to that explained previously for the PLLA/Gly preparation. The amount of PLLA-g-MA in a mixture was kept constant relative to the amount of PLLA.

\subsection{Characterization}

2.31. Fourier Transform Infrared Spectroscopy (FTIR). The molecular structure of the blend films was observed using a Spectrum Nicolet iS10 FTIR Spectrometer (Thermo Scientific). All the infrared spectra were recorded in the range of wavenumber $400-3600 \mathrm{~cm}^{-1}$.

2.3.2. Dynamic Mechanical Analysis (DMA). The mechanical properties of the pristine PLLA and blend films, consisting of tensile strength, Young's modulus, and elongation at break were measured using DMA (Mettler Toledo). The sample of $2 \mathrm{~cm} \times 0.5 \mathrm{~cm}$ (length $\times$ width) was placed between two clamps and pulled vertically with a force of $100 \mathrm{~N}$.

2.3.3. Scanning Electron Microscopy (SEM). The blend films were frozen and broken down by hand to prepare the fracture surface or interior morphology, which were then examined using an Inspect S50 Scanning Electron Microscope (SEM). The SEM samples were coated with silver prior to examination. The optimum accelerating voltage for the SEM observation was $20.00 \mathrm{kV}$ at 15,000 times magnification.

2.3.4. X-Ray Diffraction (XRD). XRD analysis was carried out by using a Panalytical X'PERT PRO XRD equipment to observe the crystallinity of the film samples. The samples were scanned in the range of $2 \theta=0-40^{\circ}$. Determination of the degree of crystallinity can be calculated using the following formula: 
$\operatorname{Crl}(\%)($ degree of crystallinity $)=(($ Total Area-Amorphous Area $)) /($ Total Area $) \times 100 \%$

2.3.5. Differential Scanning Calorimetry (DSC). The glass transition temperature $\left(\mathrm{T}_{\mathrm{g}}\right)$ of the film samples was observed using a DSC instrument (Diamond, PerkinElmer Corp., Massachusetts, USA) under a dry/inert nitrogen flow of $20 \mathrm{~cm}^{3} / \mathrm{min}$. The samples were first heated to maximum melting temperature to erase their thermal history and held for 5 minutes. Subsequently, the samples were quenched to a temperature below $\mathrm{T}_{\mathrm{g}}$ at a cooling rate of $320^{\circ} \mathrm{C} / \mathrm{min}$ for 5 minutes, followed by a second heating to a temperature above the melting temperature at a scanning rate of $20^{\circ} \mathrm{C} / \mathrm{min}$. The DSC trace of the second heating was used to characterize the $\mathrm{T}_{\mathrm{g}}$ of the samples.

\section{RESULTS AND DISCUSSION}

\subsection{Analysis of Molecular Structure and Interaction of the Blend Films}

FTIR characterization was performed to reveal the molecular structure and interaction of the composite films. Figure 1a shows the FTIR spectra of the pure glycerol, PLLA and the PLLA/Gly blend films. The spectrum of pure glycerol shows a dominant peak at wavenumber $3273 \mathrm{~cm}^{-1}$, corresponding to the peak of the hydroxyl group $(-\mathrm{OH})$, which is commonly seen in the wavenumber range of $3200-3600 \mathrm{~cm}^{-1}$. Pristine PLLA has the characteristic adsorption peak of the carbonyl group $(\mathrm{C}=\mathrm{O})$ at $\sim 1750 \mathrm{~cm}^{-1}$, as shown in Figure 1a. The adsorption peak of the carbonyl group in PLLA commonly appears at wavenumbers of 1749, 1758, 1767 and $1776 \mathrm{~cm}^{-1}$, which are assigned to some conformers of the carbonyl group (Meaurio et al., 2006). After the addition of glycerol, the FTIR spectra of the PLLA/Gly films show that the peak intensity of the hydroxyl group $(\mathrm{O}-\mathrm{H})$ increases with increasing glycerol content, indicating successful blending of glycerol and PLLA with a precise blend composition variation. The peak intensity of carbonyl groups increases with glycerol content because higher hydrogen bonding occurs between the hydroxyl group in glycerol and the carbonyl group in PLLA. The peak of the $-\mathrm{OH}$ group in the PLLA/Gly films shifts slightly compared to the corresponding peak in pure glycerol, indicating the presence of interaction between the $-\mathrm{OH}$ and $-\mathrm{C}=\mathrm{O}$ groups as a result of hydrogen bonding.

Figures $1 \mathrm{~b}, 1 \mathrm{c}$ show the FTIR spectra of the samples with the addition of compatibilizer (PLLA-g-MA). The samples without PLLA-g-MA are also displayed for comparison. Figure 1b shows the FTIR spectra of neat PLLA and PLLA-g-MA. The peak intensity of the carbonyl group in the neat PLLA-g-MA spectrum is higher than that in the neat PLLA spectrum because the structure of MA also contains the carbonyl group. Therefore, grafting MA onto PLLA results in the addition of carbonyl groups to the compound. Figure 1c show the spectra of the blend films with and without PLLA-g-MA. The spectrum of the PLLA/Gly (80/20) blend film with compatibilizer (PLLA-g-MA) shows that the peak intensity of the carbonyl group $(\mathrm{C}=\mathrm{O})$ slightly increases because of the increase in the number of carbonyl groups from the MA. Therefore, the addition of PLLA-g-MA to a PLLA blend with glycerol increases the number of interactions between the $-\mathrm{OH}$ and $-\mathrm{C}=\mathrm{O}$ groups via hydrogen bonding, which enhances the compatibility between PLLA and glycerol. The compatibility between two components in a blend strongly influences the physical properties of the new composite material, as discussed in the following section. Figure 1c also shows the slight shift in the peak of the hydroxyl group (O-H) for the [PLLA/PLLA-g-MA]/Gly (80/20) spectrum, from a wavenumber of around 3350 to $3400 \mathrm{~cm}^{-1}$. This upward shift may be caused by the interaction between the hydroxyl and carbonyl groups via the hydrogen bonding induced by higher number of carbonyl group after the addition of MA. 

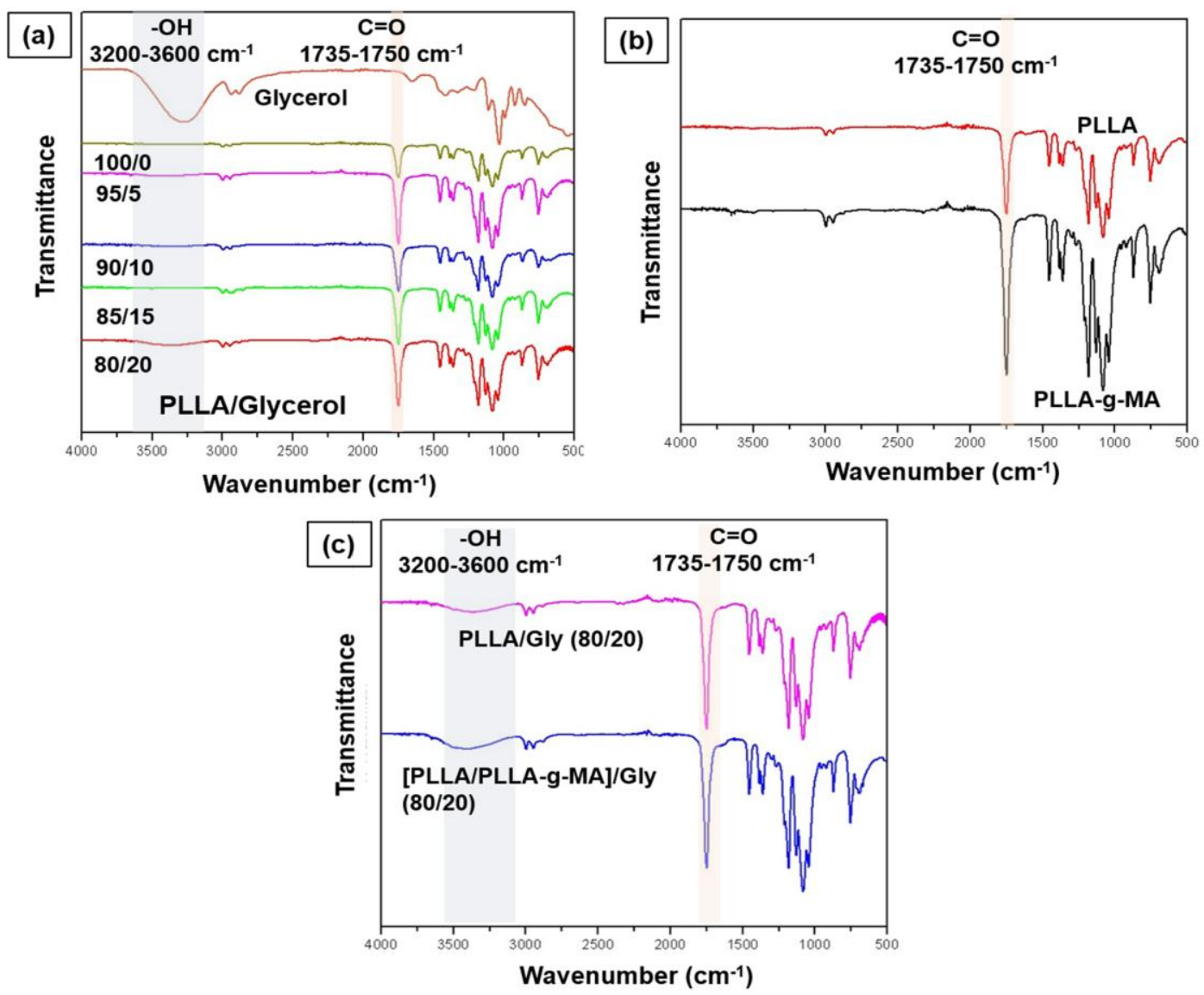

Figure 1 FTIR spectra of: (a) PLLA/Gly blend films with various variables as indicated; (b) neat PLLA and PLLA-g-MA; (c) blend films of PLLA/Gly (80/20) with and without compatibilizer

\subsection{Thermal Property Analysis}

DSC characterization was conducted to observe the effect of glycerol as an additive and PLLA g-MA as a compatibilizer on the thermal properties of the blend films. Figure 2 shows the DSC traces of neat PLLA and PLLA/Gly films with and without compatibilizer. Figure 2a displays the decrease in $\mathrm{T}_{\mathrm{g}}$ of the PLLA after the addition of glycerol. This decrease $\mathrm{T}_{\mathrm{g}}$ is proportional to the increase in glycerol content, as indicated by the arrows in Figure 2a. The decrease in $T_{g}$ shows the higher molecular chain mobility of PLLA. It can be concluded that the addition of glycerol has a plasticizing effect on PLLA. Figure $2 \mathrm{~b}$ shows a comparison of the DSC traces of the films with the addition of a compatibilizer. The $T_{g}$ of neat PLLA film with the addition of PLLA-g-MA is lower than that of the film without such an addition. The blend film of [PLLA/PLLA-g-MA]/Gly (80/20) also has slightly lower $\mathrm{T}_{\mathrm{g}}$ compared to the PLLA/Gly (80/20) film. These results show the enhancement of the compatibility between PLLA and glycerol after the addition of PLLA-g-MA, which leads to the more pronounced plasticizing effect of glycerol on the PLLA matrix. The improvement in compatibility between PLLA and glycerol also improves the interaction between the two components. 

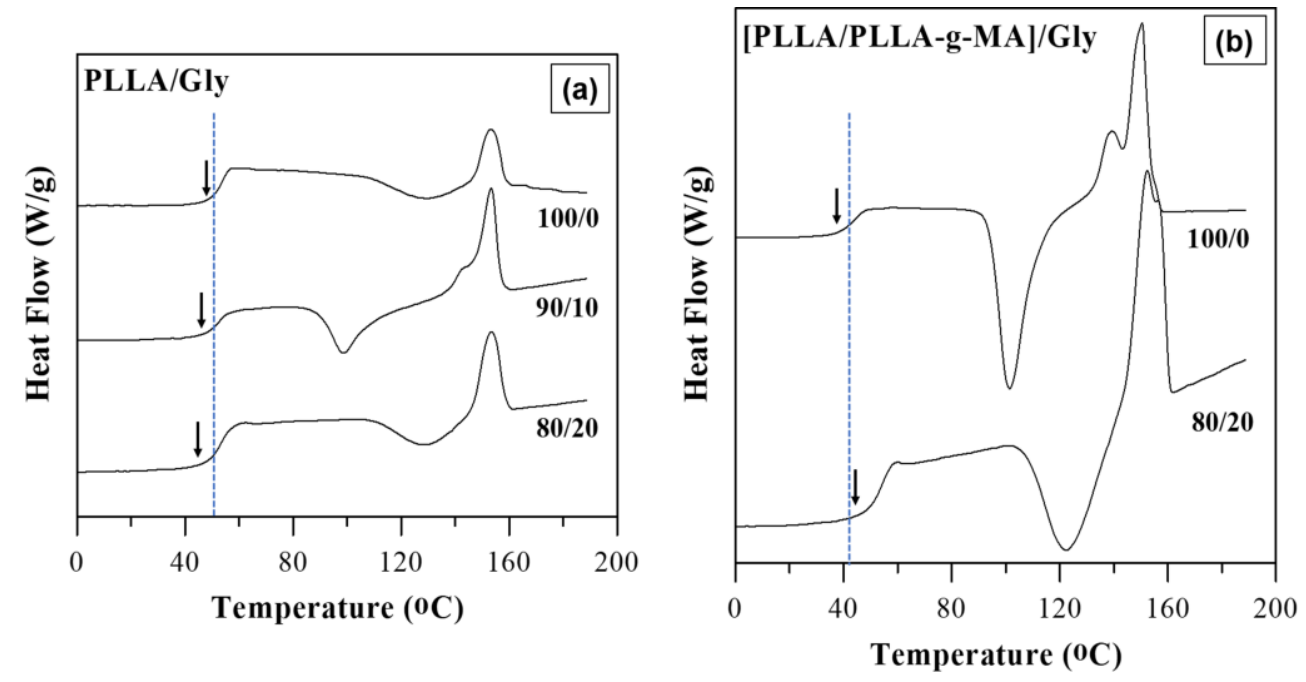

Figure 2 DSC traces of PLLA/Gly blend films: (a) without PLLA-g-MA; and (b) with PLLA-g-MA

\subsection{Mechanical Property Analysis}

Figure 3 shows the mechanical properties in terms of tensile strength, elongation at break and Young's modulus of the films without the addition of compatibilizer. The tensile strength and Young's modulus of the PLLA films decrease with increasing glycerol content. The decrease in Young's modulus shows the reduction of the films' stiffness, which means that they become more flexible. The decrease in tensile strength of the films means that the maximum force required to break them also decreases, which indicates that they become softer. On the other hand, the elongation at break of the PLLA films increases with increased glycerol content, showing that they become more elastic. Detailed values of the mechanical properties of all the films are listed in Table 1. All the results confirm that the addition of glycerol has a plasticizing effect on the PLLA matrix, and agree with the study of Sungsanit et al. (2012), which found that the plasticizer results in easier stretching and drawing.
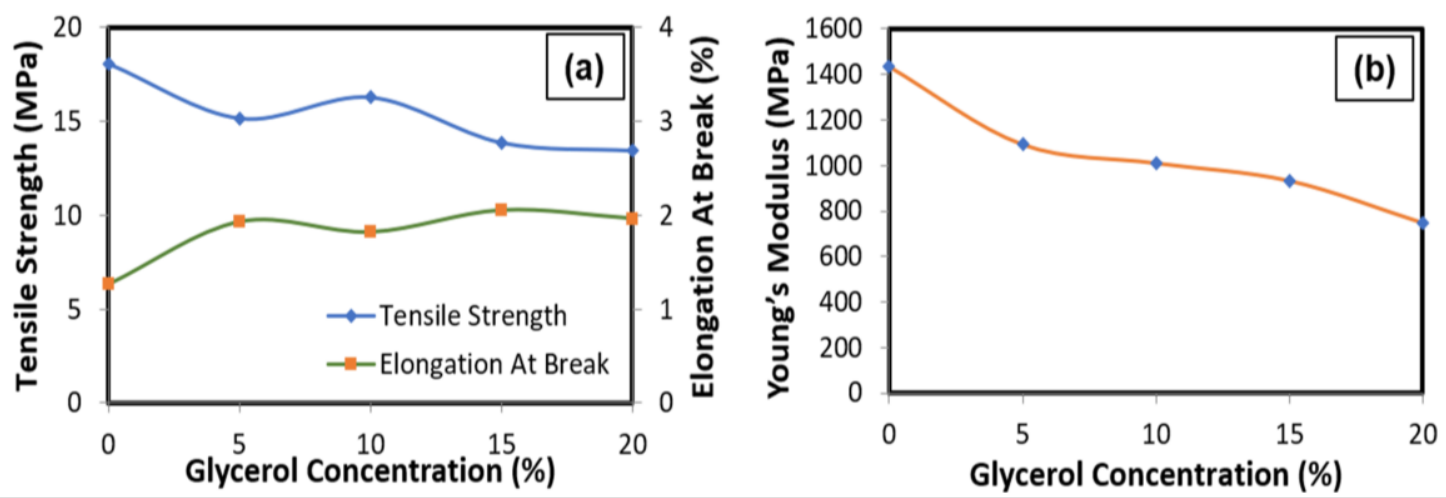

Figure 3 Tensile strength and elongation at break of the PLLA/Gly films: (a) Young's modulus of the PLLA/Gly films; (b) with variation in glycerol content

Figure 4 shows a comparison of the mechanical properties of the films with and without compatibilizer for specific compositions. The films with compatibilizer have lower tensile strength and Young's modulus, but higher elongation at break compared with those without compatibilizer. They are also softer and more flexible than those without PLLA-g-MA. Enhancement of the compatibility between PLLA and glycerol results in a more pronounced plasticizing effect of the glycerol on the PLLA matrix. The improvement in compatibility results in an increase in the interfacial bonding between the two components and enhances the 
dispersion of glycerol in the PLLA matrix, which contribute to the better mechanical properties. Similar to the blend system of polymer nanocomposites, good dispersion of nanoparticles in the polymer matrix significantly improves the properties of the nanocomposites, such as thermal degradation, compared to the pristine polymer (Abdullah et al., 2013).
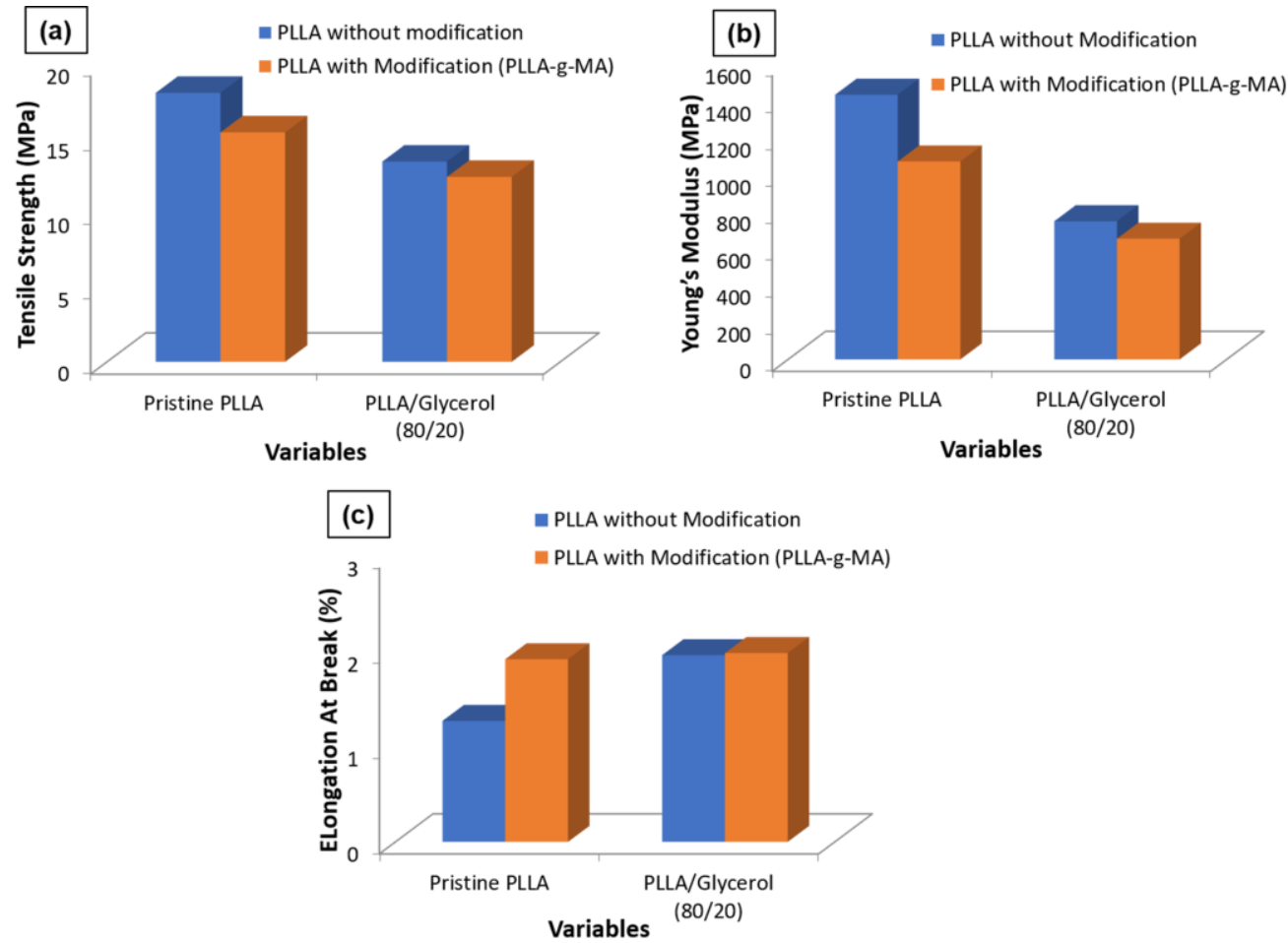

Figure 4 Comparison of the mechanical properties of the films with and without PLLA-g-MA modification: (a) tensile strength; (b) Young's modulus; (c) elongation at break

Table 1 Mechanical properties of PLLA/Gly films

\begin{tabular}{cccc}
\hline PLLA/Gly & $\begin{array}{c}\text { Tensile Strength } \\
(\mathrm{MPa})\end{array}$ & $\begin{array}{c}\text { Young's Modulus } \\
(\mathrm{MPa})\end{array}$ & $\begin{array}{c}\text { Elongation at Break } \\
(\%)\end{array}$ \\
\hline $100 / 0$ & 18.04 & 1432 & 1.27 \\
$95 / 5$ & 15.14 & 1091 & 1.93 \\
$90 / 10$ & 16.26 & 1009 & 1.82 \\
$85 / 15$ & 13.85 & 932.1 & 2.05 \\
$80 / 20$ & 13.43 & 747.8 & 1.96 \\
\hline
\end{tabular}

\subsection{Effect of the Addition of Glycerol on the PLLA Morphology}

Figure 5 shows SEM images of the fracture surface of films with various compositions. The morphology of neat PLLA film shows a smooth structure. After the addition of $10 \mathrm{wt} \%$ glycerol, the PLLA morphology shows some small pores distributed in the fracture area of the PLLA matrix. The pores become more obvious with an increase in glycerol content. Moreover, the addition of compatibilizer significantly changes the PLLA morphology, creating a porous structure homogeneously distributed in the PLLA matrix. This porous structure may be suitable as a scaffold in tissue engineering since the films contain biomaterials. It is important that further investigation related to applications in the biomedical field be conducted. 


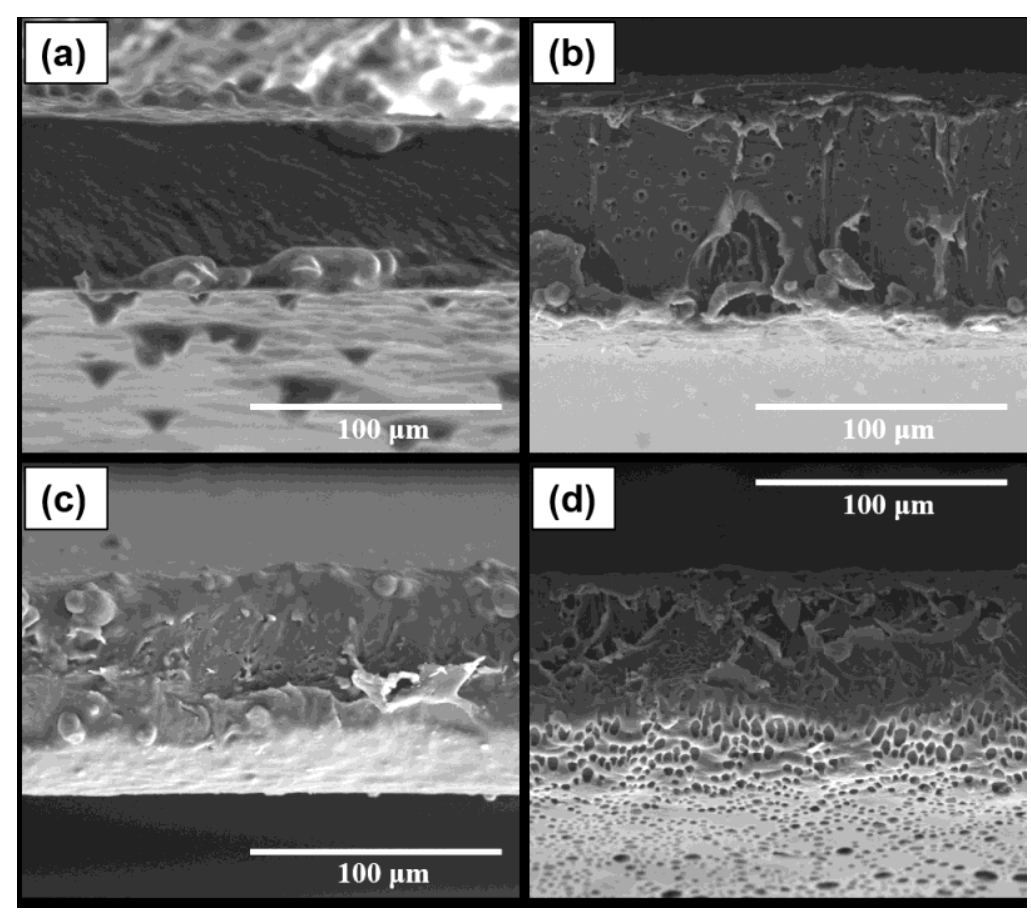

Figure 5 Fractured surface SEM images of the films: (a) neat PLLA; (b) PLLA/Gly (90/10); (c) PLLA/Gly (80/20); (d) [PLLA/PLLA-g-MA]/Gly (80/20)

\subsection{Effect of the Sddition of Glycerol on PLLA Crystallinity}

The XRD diffractograms of the blend films shown in Figure 6 show the effect of the addition of glycerol on the crystallinity of the PLLA. The peaks at $2 \theta$ of $16.5^{\circ}$ and $18.6^{\circ}$ corresponding to the PLLA crystals become sharper as the glycerol content increases, indicating higher crystallinity. Detailed values of the crystallinity of blend films without compatibilizer are listed in Table 2. The decrease in $\mathrm{T}_{\mathrm{g}}$ after the addition of glycerol enhances molecular chain mobility, meaning the chain is easily packed into crystal form when crystallization conditions occur. The blend with compatibilizer, [PLLA/PLLA-g-MA]/Gly (80/20), has a higher crystallinity index of $39.385 \%$ than the PLLA/Gly (80/20) blend film, whose index is $38.47 \%$. The addition of compatibilizer clearly enhances the compatibility of the two components, leading to improvement in the properties of the blend materials.

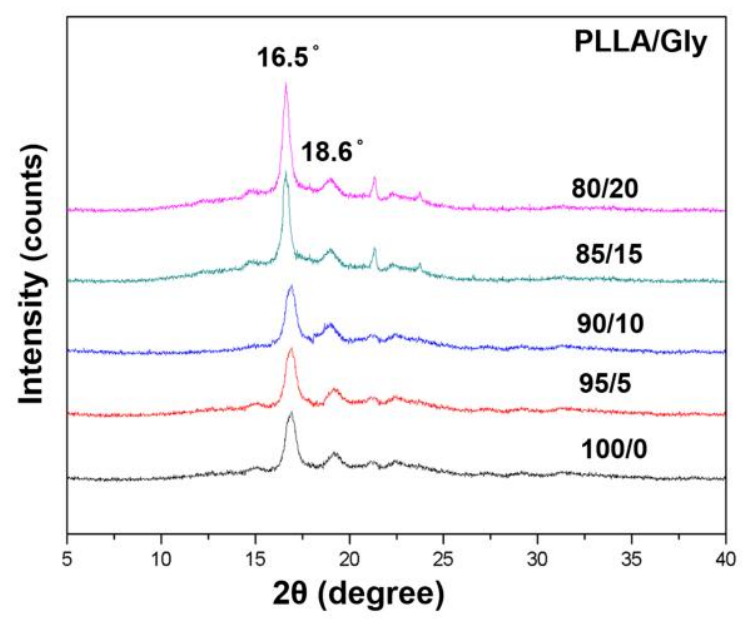

Figure 6 XRD diffractograms of blend films with varying blend compositions 
Table 2 Crystallinity index of the PLLA/Gly films

\begin{tabular}{cccc}
\hline PLLA/Gly & Total Area & Amorphous Area & Crystallinity Index, CrI (\%) \\
\hline $100 / 0$ & 126326 & 101092.3 & 19.97 \\
$95 / 5$ & 81661.33 & 57129.67 & 30.04 \\
$90 / 10$ & 113616 & 76590.67 & 32.59 \\
$85 / 15$ & 71613.67 & 47216.67 & 34.07 \\
$80 / 20$ & 54336.33 & 33430.67 & 38.47 \\
\hline
\end{tabular}

\section{CONCLUSION}

Blend films of PLLA and glycerol with and without MA modification have been prepared and characterized in term of their properties. The physical properties, including thermal and mechanical ones, and the crystallinity of the films show improvement after the addition of glycerol, and further enhancement after the addition of compatibilizer (PLLA-g-MA). The decrease in tensile strength and Young's modulus, and the increase in elongation at break for the composite films, show that they become softer and more flexible. The enhanced mechanical properties were caused by the improvement in interfacial bonding in the blend samples. Moreover, the morphology of the blend films displays a porous structure, which is similar to the characteristic three-dimensional structure of scaffolds in tissue engineering. Therefore, the biodegradable composite films with porous structures and excellent physical properties have the potential to be considered as scaffolds in tissue engineering applications, after further analysis has been conducted.

\section{ACKNOWLEDGEMENT}

This work has been financially supported by a "Penelitian Pasca Doktor (Grant Number: 503/PKS/ITS/2017)" research grant for year 2017 from DRPM, Indonesia, to which the authors express their gratitude.

\section{REFERENCES}

Abdullah, M.A.A., Mamat, M., Awang, M., Kusrini, E., Mubin, F.N.A., Sudin, N.H., 2013. Effect of Trihexyltetradecylphosphonium on Thermal Degradation Properties of Low Linear Density Polyethylene/Montmorillonite Nanocomposites. International Journal of Technology, Volume 4(2), pp. 129-135

Chen, B.Y., Jing, X., Mi, H.Y., Zhao, H., Zhang, W.H., Peng, X.F., Turng, L.S., 2015. Fabrication of Polylactic Acid/Polyethylene Glycol (PLA/PEG) Porous Scaffold by Supercritical $\mathrm{CO}_{2}$ Foaming and Particle Leaching. Polymer Engineering and Science, Volume 55(6), pp. 1339-1348

Desa, M.S.Z.M., Hassan, A., Arsad, A., Arjmandi, R., Mohammad, N.N.B., 2016. Influence of Rubber Content on Mechanical, Thermal, and Morphological Behavior of Natural Rubber Toughened Poly(Lactic Acid)-Multiwalled Carbon Nanotube Nanocomposites. Journal of Applied Polymer Science, Volume 133(48), pp. 44344-44353

Duarte, A.R.C., Mano, J.F., Reis R.L., 2010. Novel 3D Scaffolds of Chitosan-PLLA Blends for Tissue Engineering Applications: Preparation and Characterization. Journal of Supercritical Fluids, Volume 54(3), pp. 282-289

Frydrych, M., Román, S., MacNeil, S., Chen, B., 2015. Biomimetic Poly(Glycerol Sebacate)/Poly(L-Lactic Acid) Blend Scaffolds For Adipose Tissue Engineering. Acta Biomaterialia, Volume 18, pp. 40-49 
Gao, H., Xie, Y., Ou, R., Wang, Q., 2012. Grafting Effects of Polypropylene/Polyethylene Blends with Maleic Anhydride on the Properties of the Resulting Wood-Plastic Composites. Composites: Part A, Volume 43(1), pp. 150-157

Lee, L.T., Woo, E.M., Hsieh, Y.T., 2012. Macro- and Micro-Lamellar Assembly and Mechanisms for Unusual Large-Pitch Banding in Poly(L-lactic acid). Polymer, Volume 53(23), pp. 5313-5319

Meaurio, E., Lopez-Rodriguez, N., Sarasua, J.R., 2006. Infrared Spectrum of Poly(L-lactide): Application to Crystallinity Studies. Macromolecules, Volume 39(26), pp. 9291-9301

Ni'mah, H., Woo, E.M., Chang, S.M., 2014. Composite Banded Core and Non-banded Shell Transition Patterns in Stereocomplexed Poly(lactide acid) Induced by Strongly Interacting Poly(p-vinyl phenol). RSC Advances, Volume 4(99), pp. 56294-56301

Ni'mah, H., Ningrum, E.O., Sumarno, S., Rizkiyah, D.N., Chandra Divta, I.G.A.G., Meiliefiana, M., Subaghio, M.A., 2017. Effect of Particle Size and Crystallinity of Cellulose Filler on the Properties of Poly(L-lactic acid): Mechanical Property and Thermal Stability In: AIP Conference Proceedings, Volume 1840(1)

Nofar, M., Sacligil, D., Carreau, P.J., Kamal, M.R., Heuzey, M.C., 2019. Poly(Lactic Acid) Blends: Processing, Properties and Applications. International Journal of Biological Macromolecules, Volume 125, pp. 307-360

Pavia, F.C., Rigogliuso, S., La Carrubba, V., Mannella, G.A., Ghersi, G., Brucato, V., 2012. Poly Lactic Acid Based Scaffolds for Vascular Tissue Engineering. Chemical Engineering Transaction, Volume 27, pp. 409-414

Rai, R., Tallawi, M., Grigore, A., Boccaccini, A.R., 2012. Synthesis, Properties and Biomedical Applications of Poly(glycerol sebacate) (PGS): A Review. Progress in Polymer Science, Volume 37(8), pp. 1051-1078

Raquez, J.M., Habibi, Y., Murariu, M., Dubois, P., 2013. Polylactide (PLA)-based Nanocomposites. Progress in Polymer Science, Volume 38(10-11), pp. 1504-1542

Saini, P., Arora, M., Ravi Kumar, M.N.V., 2016, Poly(lactic acid) Blends in Biomedical Applications. Advanced Drug Delivery Reviews, Volume 107, pp. 47-59

Sitompul, J.P., Insyani, R., Prasetyo, D., Prajitno, H., Lee, H.W., 2016. Improvement of Properties of Poly(L-lactic acid) through Solution Blending of Biodegradable Polymers. Journal of Engineering and Technological Sciences, Volume 48(4), pp. 430-441

Sungsanit, K., Kao, N., Bhattacharya, S.N., 2012. Properties of Linear Poly(Lactic Acid)/Polyethylene Glycol Blends. Polymer Engineering and Science, Volume 52(1), pp. $108-116$

Wang, H.M., Chou, Y.T., Wu, C.S., Yeh, J.T., 2012. Polyester/Cellulose Acetate Composites: Preparation, Characterization and Biocompatible. Journal of Applied Polymer Science, Volume 126(S2), pp. E242-E251

Wang, Y., Ameer, G.A., Sheppard, B.J., Lange, R., 2002. A Tough Biodegradable Elastomer. Nature Biotechnology, Volume 20, pp. 602-606

Woo, E.M., Ni'mah, H., Wang, Y.H., 2014. Anisotropic Nucleation and Janus-Faced Crystals of Poly(L-lactic acid) Interacting with an Amorphous Diluent. Industrial and Engineering Chemistry Research, Volume 53(23), pp. 9772-9780

Zhang, J.F., Sun, X., 2004. Mechanical Properties of Poly(Lactic Acid)/Starch Composites Compatibilized by Maleic Anhydride. Biomacromolecules, Volume 5(4), pp. 1446-1451 